Background Non-consensual sexual activity between young people continues to be a major public health problem with long-term negative health outcomes. To our knowledge no study has yet evaluated violation and rape separately in girls, boys and boys who have sex with men (MSM).

Methods Data were obtained from 'sex under the age of 25', a large national study on sexual behaviour and sexual health of 10,404 young people (12-25 years), Netherlands. Current analyses were restricted to sexually experienced young people $(n=6,200)$. Multinomial regression was used to examine associations for ever experienced (1) violation (unwanted kissing and touching) or (2) rape (vaginal or anal penetration by finger, penis or mouth) compared to consensual sex. Evaluated determinants included sexual behaviour, drug use, social network and attitude. Analyses were stratified for girls ( $n=3779)$, boys $(n=2209)$ and MSM $(n=212)$ and controlled for age, educational level and ethnicity.

Results Violation was reported by $29.6 \%$ of sexually experienced girls, $20.6 \%$ of boys, and $22.6 \%$ of MSM. Rape was reported by $24.2 \%, 4.4 \%$ and $14.2 \%$ in the three groups, respectively. Rape was significantly associated with a negative attitude to porn (in girls, boys, MSM), receiving non-monetary goods in exchange for sex (girls, boys), use of alcohol and drugs (girls), sex on the internet (girls, boys), talking to friends about sex (MSM), sexual problems (girls, boys), negative self image (girls), multiple sex partners (girls, boys, MSM), early sexual debut (girls) and low self efficacy (girls). All these factors except porn were also associated with violation, although associations were sometimes observed in different groups than for rape.

Conclusion The prevalence of non-consensual sex is alarmingly high in young people who have experience with sex. Girls and young MSM boys appear to be especially vulnerable. Healthcare providers and educators should continue to address these issues in care and education.

\section{P4.066 SEXUAL HEALTH OF OLDER MEN ATTENDING A GENITO- URINARY MEDICINE CLINIC}

doi:10.1136/sextrans-2013-051184.0964

E C Wainwright, J Sherrard. Genito-urinary Medicine Department, Oxford University Hospitals NHS Trust, Oxford, UK

Many healthcare professionals, particularly non-specialists, do not consider older men to be at risk of sexually transmitted infections (STIs). Recent Health Protection Agency data show that the percentage of STIs in older age groups in the UK is increasing and are considerably higher for men than women. Men who have sex with men (MSMs) remain at high risk of STIs throughout the age groups.

There is a scarcity of published data on older men attending genito-urinary medicine (GUM) clinics. This project aimed to review men aged 50 and over presenting to a GUM outpatient clinic in the first 3 months of 2012 .

4056 men attended during the 3 months, of whom 105 (3\%) were 50 years and older. Records were available for 102 men. The age range was 50 to 90 years (mean 58; SD 7.2) and 31 (30\%) were MSMs.

$12 / 31$ (39\%) MSMs and 35/71 (49\%) heterosexuals reported that they never use condoms. 7/31 (23\%) MSMs and 7/71 (10\%) heterosexuals were married to women. A significantly higher proportion of MSM-episodes $(17 / 31 ; 55 \%)$ reported $>1$ partner in the preceding 3 months compared to heterosexual-episodes (17/72; $24 \%$; P value 0.004). An STI was diagnosed in 6/31 (19\%) MSM and 23/72 (32\%) heterosexuals: including non-specific urethritis, proctitis, epididymo-orchitis, warts, gonorrhoea, chlamydia, herpes, syphilis, scabies, pubic lice, HIV and hepatitis B.

This case note review found significant numbers of older men presenting to the GUM clinic. There was a high prevalence of STIs.
A surprisingly high proportion of MSMs were married, which suggests that, if they were to attend a non-specialist setting, it is unlikely they would be identified as being at risk of STIs.

Many people remain sexually active into their 80 s and beyond. These data support the need to challenge preconceptions regarding sexual health and STI risk in older men.

\section{P4.067 PHOTOVOICE}

doi:10.1136/sextrans-2013-051184.0965

\section{S Lex. Aids Hilfe Wien, Vienna, Austria}

Background In Austria, Sub-Saharan African migrants are disproportionately affected by HIV. Since HIV is also a social issue and its transmission is often conditioned by vulnerability, we wanted to look on the following questions from a broader point of view:

- Which factors support my integration in Austria?

- Which factors hinder my integration in Austria?

These questions seemed relevant in order to get to know a target group's life situation and to be able to adapt preventive measures to it. Method The method used to answer these questions was Photovoice - a participatory visual method for a critical questioning of social structures. It evolved from participatory research and should enhance communities to share their experiences and to work collaboratively on change. It also gives insights into how they conceptualise their circumstances and their hopes for the future. Photovoice also wants to bring about positive change into communities through providing them with photographic training.

Implementation Six people from sub-Saharan African countries were invited to five workshops, led by a professional photographer and the project leader. During the workshops, basic techniques of photography were taught and participants were asked to take pictures according to the research questions. Together with the group the taken pictures were discussed together, narratives to go with their photos were developed, and conducting outreach or other action was reviewed.

Outcome The product of the project is a booklet telling the storeys of the participants together with their taken pictures. The booklet will be shared with various organisations and politicians. The gotten information will be integrated in existing prevention projects among the sub-Saharan African communities in Vienna.

\section{P4.068 HAZARDOUS ALCOHOL CONSUMPTION AND SEXUAL PARTNER CONCURRENCY AMONG ADULTS IN RURAL SOUTH AFRICA}

doi:10.1136/sextrans-2013-051184.0966

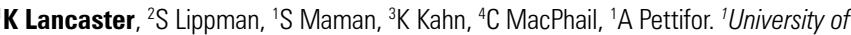
North Carolina, Chapel Hill, Chapel Hill, NC, United States; ${ }^{2}$ Center for AIDS Prevention Studies (CAPS), UCSF, San Francisco, CA, United States; 'Wits University Rural Public Health and Health Transitions Research Unit, Agincourt, South Africa; ${ }^{4}$ Wits Reproductive Health and HIV Institute (WRHI), Johannesburg, South Africa

Objectives Sexual partner concurrency and hazardous alcohol consumption patterns both lead to accelerated transmission of HIV/STIs. While there has been considerable research undertaken documenting the correlation of alcohol and HIV risk behaviours, there is less research examining the association of hazardous and harmful drinking patterns and concurrent sexual relationships.

Methods A 2012 cross section of 483 (49\%) males and 501 (51\%) females, aged 18-35 years, in rural Bushbuckridge, South Africa, were surveyed. Hazardous and harmful patterns of alcohol consumption were identified using Alcohol Use Disorders Identification Test (AUDIT) score $\geq 8$. Having concurrent sexual partners was determined as more than one ongoing, sexual partnership reported on the day of the interview. Prevalence ratios were estimated using 